Article

\title{
Synthesis, DNA Binding, and Anticancer Properties of Bis-Naphthalimide Derivatives with Lysine-Modified Polyamine Linkers
}

\author{
Yu Huang ${ }^{1, *}$, Chun-Xia Wu ${ }^{1}$, Yu Song ${ }^{1}$, Min Huang ${ }^{2}$, Da-Nian Tian ${ }^{2}$, Xin-Bin Yang ${ }^{3}$ \\ and Yan-Ru Fan 1,* \\ 1 Key Laboratory of Hui Ethnic Medicine Modernization, Ministry of Education, \\ Ningxia Engineering and Technology Research Centre of Hui Medicine Modernization, \\ College of Pharmacy, Ningxia Medical University, Yinchuan 750004, China; \\ wuchunxiapharmacy@163.com (C.-X.W.); songyuouc@163.com (Y.S.) \\ 2 College of Public Health, Ningxia Medical University, Yinchuan 750004, China; \\ huangmin@163.com (M.H.); tiandanian@163.com (D.-N.T.) \\ 3 Rongchang Campus, Southwest University, Chongqing 402460, China; yangxbqq@126.com \\ * Correspondence: huangyu@nxmu.edu.cn (Y.H.); risefyr@163.com (Y.-R.F.); \\ Tel.: +86-951-688-0693 (Y.H. \& Y.-R.F.)
}

Received: 28 December 2017; Accepted: 16 January 2018; Published: 29 January 2018

\begin{abstract}
A series of bis-naphthalimide derivatives with different diamine linkers were designed and synthesized. All of the synthesized bis-naphthalimide derivatives were characterized by NMR and HRMS spectra. The binding ability between the compounds and CT DNA was evaluated by using UV-Vis titration experiments. The bis-naphthalimide compound with an ethylenediamine linker showed the largest binding constant with CT DNA. Hence, it was used as the model compound to study the DNA binding selectivity by UV-Vis titration aiming at different DNA duplexes. As a result, this compound showed binding preference to AT-rich duplexes. The DNA binding modes of the compounds were also measured by viscosity titration. The cytotoxicity of the compounds was evaluated by MTT assay. Compounds with 1,6-diaminohexane or 1,4-phenylenedimethanamine linkers showed higher cytotoxicity compared with other bis-naphthalimide derivatives.
\end{abstract}

Keywords: bis-naphthalimide derivatives; DNA binding; cytotoxicity

\section{Introduction}

Naphthalimide (1H-benzo[de]isoquinoline-1,3-(2H)-diones), a kind of flat heteroaromatic polycyclic amide, has been studied for decades due to its potential for the development of antitumor drugs [1]. Though some of its derivatives were approved for clinical trials, all of the trials were terminated due to the toxic side effects [2-7]. Therefore, to improve the antitumor activity and reduce the side effects, modifications on the naphthalimide structure have been carried out in recent decades; some naphthalimide derivatives with different side chains, aromatic ring systems, and substituents on the ring have been designed and synthesized [8-11].

Dimerization of naphthalimide is one of the generally used methods to improve its antitumor efficiency. The dimeric naphthalimide derivatives usually exhibit higher activity than the monomeric ones. The bis-naphthalimide Elinafide, which exhibits high activity against a series of human xenograft models, is a well-known bis-intercalator in all the naphthalimide derivatives [12]. Dimerization has also been employed to link the two pharmacophores [13]. Among all types of spacers, besides Tröger's base moieties [14,15], polyamine spacers have been widely developed in the construction of bis-intercalators which exhibited high DNA binding ability and excellent anticancer activity [16-20]. Some other studies in the literature also reported the influence of bis-naphthalimide derivatives with polyamine 
spacers on DNA [21-23]. Lin et al. reported a bis-naphthalimide derivative linked by spermidine, which showed an $\mathrm{IC}_{50}$ value of 0.15 and $1.64 \mu \mathrm{M}$ towards Caco-2 and HT-29 colon adenocarcinoma cells and induced significant DNA damage [24]. Other results from Li and co-workers showed that an $N$-(2-hydroxyethyl)piperazine-modified bis-naphthalimide derivative showed better cytotoxic activity than the control drug, amonafide [25]. This compound also exhibited fluorescence enhancement upon binding with DNA.

To improve the anticancer ability, amino acids with chiral side chains were combined into the structure of naphthalimide derivatives. Qian et al. reported some naphthalimide derivatives with flexible leucine side chains which exhibited the $\mathrm{IC}_{50}$ values of $10^{-6}-10^{-5} \mathrm{M}$ against HeLa, A549, P388, HL-60, MCF-7, HCT-8, and A375 cancer cell lines in vitro [26]. In addition, the chirality of the amino side chains is important for the naphthalimide derivatives. Yang et al. reported that the S-enantiomers showed better DNA binding activity and DNA photocleavage ability than did the R-enantiomers [27].

Herein, we report the synthesis of novel bis-naphthalimide derivatives modified by lysine and different diamine linkers. The DNA binding ability and cytotoxicity of these compounds were systematically studied. All of the compounds exhibited high DNA binding ability and showed binding preference to AT-rich (adenine and thymine) duplexes. However, the cytotoxicity of these compounds was not so good.

\section{Results and Discussion}

\subsection{Chemistry}

All of the bis-naphthalimide derivatives were synthesized from 1,8-naphthalic anhydride as shown in Scheme 1. Firstly, 1,8-naphthalic anhydride was refluxed with L-lysine in ethanol to obtain compound 1. Then, compound 1 was reacted with di-tert-butyl dicarbonate to protect the amino group. After that, the carboxyl group was condensed with different diamines by a DCC (Dicyclohexylcarbodiimide) coupling reaction. Finally, the bis-naphthalimide derivatives were obtained by deprotection of the Boc group.

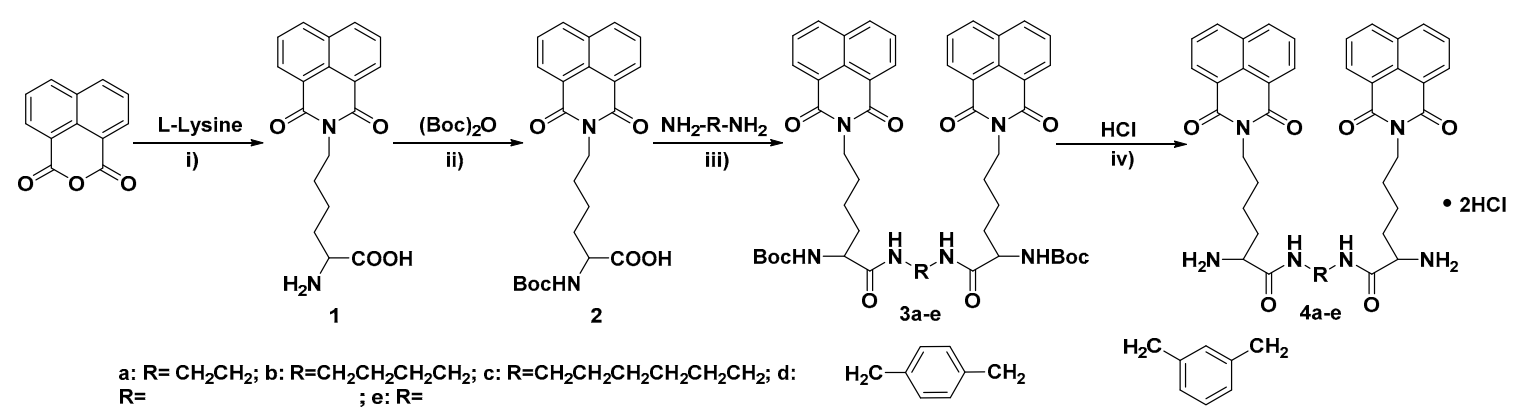

Scheme 1. The synthesis route of bis-naphthalimide derivatives. Reagents and conditions: (i) ethanol, reflux $30 \mathrm{~h}$; (ii) $\mathrm{Na}_{2} \mathrm{CO}_{3}, \mathrm{THF} /$ Water (2:1), room temperature; (iii) triethylamine $\left(\mathrm{Et}_{3} \mathrm{~N}\right.$ ), 4-dimethylaminopyridine (DMAP), 1-hydroxybenzotriazole (HOBt), dicyclohexylcarbodiimide (DCC), dichloromethane; (iv) ethanol.

\subsection{UV-Vis Titration}

According to our laser scanning confocal microscopy experiments of other bis-naphthalimide derivatives, some of these active compounds reached the nucleus of the cell (unpublished results). Hence, it was necessary to evaluate the DNA binding ability of the synthesized bis-naphthalimide derivatives $4 \mathbf{a}-\mathbf{e}$. The DNA binding abilities of the bis-naphthalimide derivatives and mononaphthalimide derivative 10 were evaluated by UV-Vis titration experiments, which was a useful method to calculate the DNA binding constants in vitro. After Calf Thymus DNA (CT DNA) was added to the phosphate buffer solution containing different compounds, all of the solutions exhibited a slight hypochromism without 
any significant spectral shift. The binding constants of the compounds were calculated by using a plot of $D / \Delta \varepsilon_{a p p}$ versus $D$, as shown in Figure 1 and Table 1 . Bis-naphthalimide derivative 4a with the shortest linker showed the largest binding constant $\left(3.40 \times 10^{4} \mathrm{~L} / \mathrm{mol}\right)$. The binding constants decreased with the linker length from ethylenediamine, to 1,4-butanediamine, to 1,6-hexanediamine. Compounds $4 \mathbf{d}$ and $4 \mathbf{e}$ with rigid bis(aminomethyl)benzene linkers were the weakest DNA binding reagents. The rigidity and length of the linkers influenced the binding ability of the bis-naphthalimide derivatives.

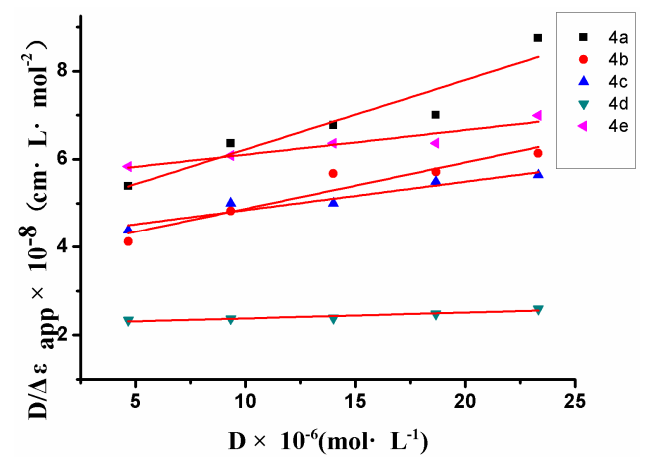

Figure 1. $D / \Delta \varepsilon_{a p p}$ vs. $D$ plot of bis-naphthalimide derivative $4(50 \mu \mathrm{M})$ with CT DNA in PBS buffer $(50.0 \mathrm{mM}, \mathrm{pH}=7.4)$.

Table 1. The $K_{b}$ values of compounds $4 \mathbf{a}-\mathbf{e}$ with CT DNA.

\begin{tabular}{cc}
\hline Compound & $\boldsymbol{K}_{\boldsymbol{b}}\left(\mathbf{M}^{-\mathbf{1}}\right)$ \\
\hline $\mathbf{4 a}$ & $3.40 \times 10^{4}$ \\
$\mathbf{4 b}$ & $2.78 \times 10^{4}$ \\
$\mathbf{4} \mathbf{c}$ & $1.54 \times 10^{4}$ \\
$\mathbf{4 d}$ & $7.29 \times 10^{3}$ \\
$\mathbf{4 e}$ & $1.00 \times 10^{4}$ \\
\hline
\end{tabular}

\subsection{DNA Binding Selectivity}

As compound 4a exhibited the largest DNA binding ability among all of the bis-naphthalimide derivatives, it was used as the model compound to study the DNA binding selectivity. The phosphate buffer solution of compound 4a was titrated using four different DNA duplexes: [Poly(dA)-Poly(dT)], [Poly(dG)-Poly(dC)], AT Box [Poly(dTATAAT)-Poly(dATATTA)], and GC Box [Poly(dGGGCGG)-Poly(dCCCGCC)], respectively. The $D / \Delta \varepsilon_{a p p}$ vs. $D$ plot of the model compound was recorded, as shown in Figure 2. The binding constant of compound 4a with different DNA duplexes was calculated. As shown in Table 2, the bis-naphthalimide derivative 4 a showed a tendency to bind with AT-rich duplexes. The binding constants of 4a with AT-rich duplexes [Poly(dA)-Poly(dT)] and AT Box were higher than those with the reference duplexes [Poly (dG)-Poly(dC)] and GC Box. The binding constant of the hybrid AT box was similar to that of [Poly(dA)-Poly(dT)], which meant that the compound 4a preferred to bind with AT-rich duplexes, no matter how hybridized the AT-rich duplexes were. The binding selectivity of the bis-naphthalimide derivatives was in accordance with similar compounds [28].

Table 2. $K_{b}$ values for compound $4 \mathbf{a}$ with various DNA sequences.

\begin{tabular}{cc}
\hline DNA Duplex & $\boldsymbol{K}_{\boldsymbol{b}}\left(\mathbf{M}^{-\mathbf{1}} \mathbf{)}\right.$ \\
\hline$[$ Poly(dA)-Poly(dT)] & $9.08 \times 10^{3}$ \\
[Poly(dG)-Poly(dC)] & $1.15 \times 10^{3}$ \\
AT Box & $9.71 \times 10^{3}$ \\
GC Box & $1.91 \times 10^{3}$ \\
\hline
\end{tabular}




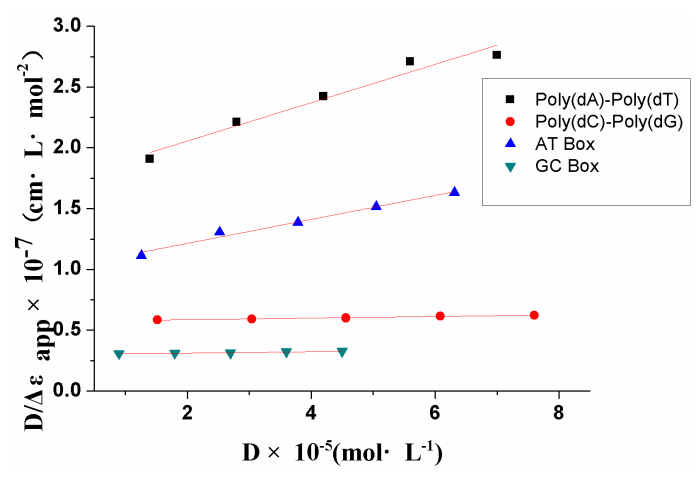

Figure 2. $D / \Delta \varepsilon_{a p p}$ vs. $D$ plot of bis-naphthalimide derivative $4 \mathbf{a}(50 \mu \mathrm{M})$ with different DNA duplexes in PBS buffer (50.0 mM, pH = 7.4).

\subsection{Viscosity Studies}

Although the UV-Vis titration data provided necessary information on the binding ability of bis-naphthalimide derivative, the detailed binding mode cannot be given by generally used optical photophysical methods. A viscosity experiment is a useful method and can provide critical information on different binding modes. Generally speaking, in classical intercalation binding mode, the intercalators insert into the base pairs of the DNA duplexes and extend the length of DNA, which significantly increases the viscosity of the DNA solution [29]. In partial and/or nonclassical intercalation binding mode, the length of DNA duplexes is reduced, which induces a decrease in the viscosity of the DNA solution [30]. As shown in Figure 3, the viscosity of the DNA solution increased after the addition of bis-naphthalimide derivatives $4 a$ and $4 b$ but decreased after the addition of compounds $4 \mathbf{c}-\mathbf{e}$ and $\mathbf{1}$. These results showed that compounds $4 \mathrm{a}$ and $4 \mathrm{~b}$ were classical DNA intercalators. Compounds $\mathbf{4} \mathbf{c}-\mathbf{e}$ and $\mathbf{1}$ bind with DNA through the partial intercalation binding mode. The rigidity of the linker significantly influenced the binding modes of the different bis-naphthalimide derivatives. To our surprise, the mononaphthalimide compound $\mathbf{1}$ exhibited a partial intercalation binding mode, which might be due to the electrostatic binding of the amino group interrupting the intercalation of the naphthalimide group. The weak binding of compound $\mathbf{4 c}$ might have the same cause.

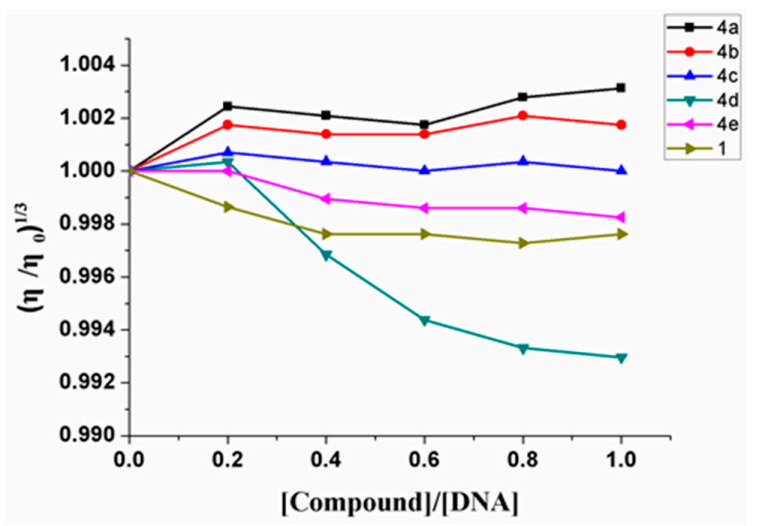

Figure 3. DNA viscosity titration of different bis-naphthalimide compounds $4 \mathbf{a}-\mathbf{e}$ at $35{ }^{\circ} \mathrm{C}$ in $25 \mathrm{~mL}$ phosphate buffer $(50.0 \mathrm{mM}, \mathrm{pH}=7.4)$. The concentration of DNA was $0.56 \mathrm{mM}$ and the ratios between bis-naphthalimide compounds and DNA were $0.2,0.4,0.6,0.8$, and 1.0 , respectively.

\subsection{Cytotoxicity Assay}

The cytotoxicity of bis-naphthalimide derivatives against human cancer cell lines EC109 and BGC823 were evaluated by MTT assay (Table 3). 5-Fluorouracil was tested as the reference compound. After the 
EC109 cell line and bis-naphthalimide derivatives were incubated for $24 \mathrm{~h}$, compounds $4 \mathrm{c}$ and $4 \mathbf{d}$ (with longer linkers) showed higher cytotoxicity compared with other bis-naphthalimide derivatives. Hence, compounds $4 \mathrm{c}$ and $4 \mathbf{e}$ were used to test the cytotoxicity against the BGC823 cell line. According to the results, the bis-naphthalimide derivative $4 \mathbf{e}$ showed moderate antitumor ability with an $\mathrm{IC}_{50}$ value of $77.99 \mu \mathrm{mol} / \mathrm{L}$ toward the BGC823 cell line, which was lower than that of 5-Fluorouracil.

Table 3. The cytotoxicity of compounds $4 a-\mathbf{e}$.

\begin{tabular}{ccccccc}
\hline \multirow{2}{*}{ Cell Lines } & \multicolumn{7}{c}{ IC $_{\mathbf{5 0}}$ Value $\left(\mu \mathbf{m o l} \cdot \mathbf{L}^{-\mathbf{1}}\right)$} \\
\cline { 2 - 7 } & Fluorouracil & $\mathbf{4 a}$ & $\mathbf{4 b}$ & $\mathbf{4 c}$ & $\mathbf{4 d}$ & $\mathbf{4 e}$ \\
\hline EC109 & 45.82 & 694.59 & 456.35 & 330.38 & 142.45 & 354.48 \\
BGC823 & 49.88 & N.T. ${ }^{1}$ & N.T. & 193.65 & 77.99 & N.T. \\
\hline \multicolumn{7}{c}{ 1 N.T.: not tested. }
\end{tabular}

\subsection{Morphology Observation}

According to the results of the MTT assay, compound 13d was used to study the morphological alterations of the BGC823 cell line in the absence and presence of bis-naphthalimide derivatives. In the absence of compound $\mathbf{4 d}$, the cancer cell line showed adherent growth with normal size and shape (Figure 4A). When cancer cells were incubated with compound $\mathbf{4 d}$, the cell counts dropped with the increasing concentration of compound $4 \mathbf{d}$ (Figure $4 \mathrm{~B}-\mathrm{F})$. The cancer cells became small and round at higher drug concentrations.
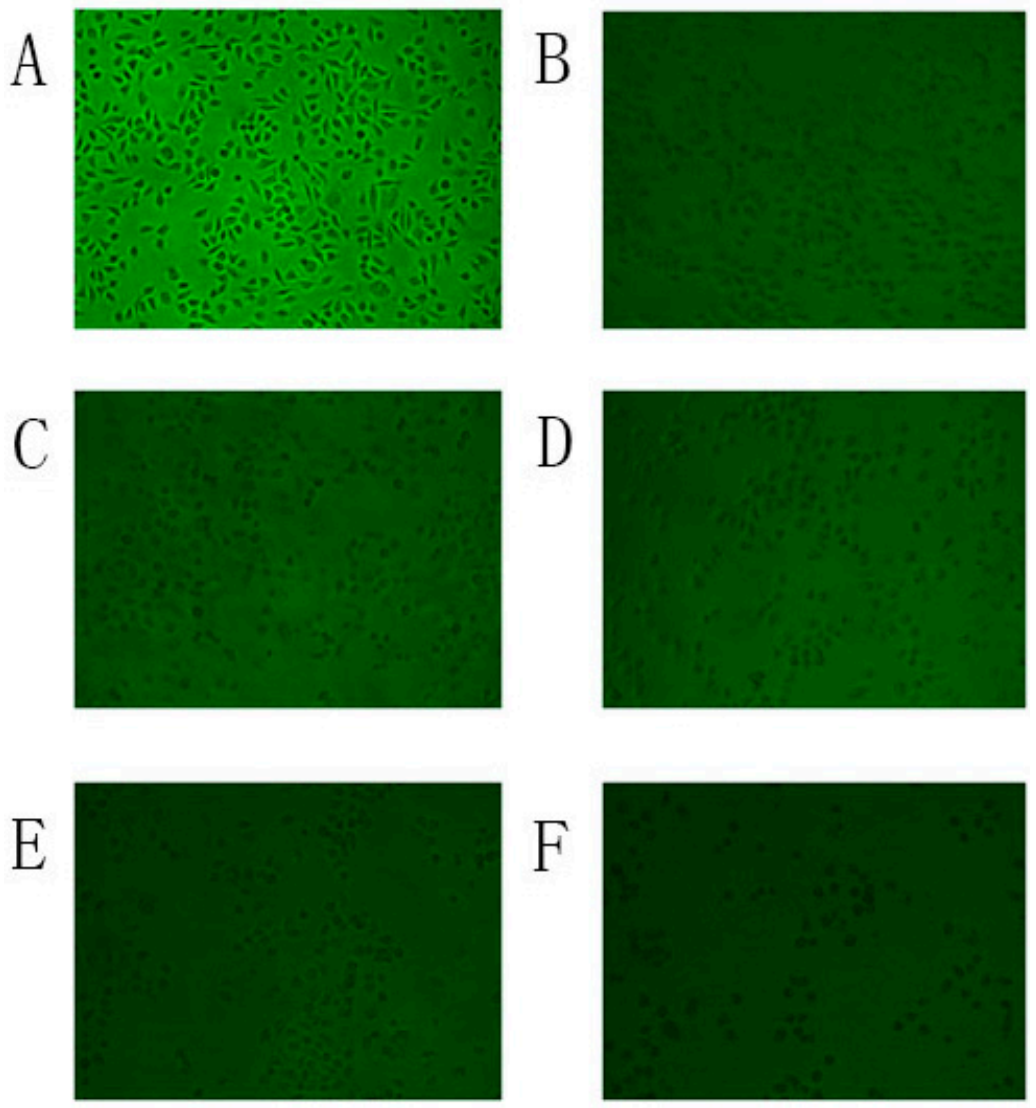

Figure 4. Morphological changes in BGC823 cells. The cells were treated with 4d (A) Control; (B) $12.5 \mu \mathrm{M}$; (C) $25 \mu \mathrm{M}$; (D) $50 \mu \mathrm{M}$; (E) $100 \mu \mathrm{M}$; (F) $200 \mu \mathrm{M}$ for $48 \mathrm{~h}$ and observed under a phase contrast microscope. 


\section{Materials and Methods}

\subsection{Chemistry}

All of the chemical reagents and solvents were of analytic grade and obtained from commercial sources. CT DNA were from Sigma-Aldrich (St. Louis, MO, USA) and other DNA duplexes were from Sangon Biotech (Shanghai, China). UV-Vis spectra were measured on a Shimadzu UV-2501 spectrophotometer (Kyoto, Japan) at room temperature. ${ }^{1} \mathrm{H}-\mathrm{NMR}$ and ${ }^{13} \mathrm{C}-\mathrm{NMR}$ spectra were recorded on a Bruker AVIII 400 spectrometer (Fällanden, Switzerland), with tetramethylsilane (TMS) as an internal standard. Mass spectra were recorded on a Shimadzu LCMS-IT-TOF (Kyoto, Japan).

\subsubsection{Synthesis of $N$-Epsilon-1,8-naphthalimido-lysine (1)}

1,8-Naphthalic anhydride $(5.00 \mathrm{~g}, 25.23 \mathrm{mmol})$ was dissolved in $500 \mathrm{~mL}$ of ethanol. After the addition of L-Lysine $(6.92 \mathrm{~g}, 37.88 \mathrm{mmol})$, the mixture was refluxed for $30 \mathrm{~h}$. Then, the insoluble solid was removed by filtration while the solvent was hot. Another $500 \mathrm{~mL}$ of ethanol was added into the filtrate and the mixture was stored overnight. After filtering the insoluble solid, the ethanol was removed under reduced pressure to obtain the crude product. Hot water was added to the crude product, and the mixture solution was then stirred for $5 \mathrm{~min}$. After the insoluble solid was filtered, the filtrate was cooled to room temperature. A yellow crystal crystallized from the water solution. The yield of $N$-epsilon-1,8-naphthalimido-lysine (1) was $60.0 \% .{ }^{1} \mathrm{H}-\mathrm{NMR}\left(400 \mathrm{MHz}, \mathrm{D}_{2} \mathrm{O}\right)$ $\delta: 8.14(\mathrm{~d}, J=7.2 \mathrm{~Hz}, 2 \mathrm{H}$, naphthalene-H), $7.88(\mathrm{~d}, J=8.0 \mathrm{~Hz}, 2 \mathrm{H}$, naphthalene- $\mathrm{H}), 7.45(\mathrm{t}, J=7.8 \mathrm{~Hz}$, $2 \mathrm{H}$, naphthalene- $\mathrm{H}), 5.40\left(\mathrm{q}, J=5.32 \mathrm{~Hz}, 1 \mathrm{H}, \mathrm{NH}_{2} \mathrm{CH}\right), 3.06\left(\mathrm{t}, J=7.6 \mathrm{~Hz}, 2 \mathrm{H}, \mathrm{NCH}_{2}\right), 1.91-2.31(\mathrm{~m}, 2 \mathrm{H}$, $\left.\mathrm{CHCH}_{2}\right), 1.68-1.77\left(\mathrm{~m}, 2 \mathrm{H}, \mathrm{NCH}_{2} \mathrm{CH}_{2}\right), 1.31-1.45\left(\mathrm{~m}, 2 \mathrm{H}, \mathrm{CHCH}_{2} \mathrm{CH}_{2}\right) .{ }^{13} \mathrm{C}-\mathrm{NMR}\left(100 \mathrm{MHz}, \mathrm{D}_{2} \mathrm{O}\right)$ $\delta: 175.66,165.25,135.05,131.48,130.60,126.81,120.57,62,46,54.70,39.21,28.63,26.50,23.19$.

\subsubsection{Synthesis of $N$-Alpha-(tert-Butoxycarbonyl)- $N$-epsilon-1,8-naphthalimido-lysine (2)}

To $300 \mathrm{~mL}$ of a THF/water (2:1) mixture solution of $N$-epsilon-1,8-naphthalimido-lysine (1) $(6.60 \mathrm{~g}$, $20.26 \mathrm{mmol})$, sodium carbonate ( $38.50 \mathrm{~g}$ ) was added. The mixture solution was stirred in an ice-water bath until the temperature was cooled to $0{ }^{\circ} \mathrm{C}$. Then, $30 \mathrm{~mL}$ THF solution of di-tert-butyl dicarbonate $(4.85 \mathrm{~g}, 22.27 \mathrm{mmol})$ was added dropwise into the flask. After the mixture was stirred overnight at room temperature, the mixture was filtered and the filtrate was condensed under reduced pressure to obtain a water solution. The water solution was extracted by ethyl acetate $(60 \mathrm{~mL})$ three times. Then, the water solution was adjusted to $\mathrm{pH} 3$ and extracted by methylene chloride $(90 \mathrm{~mL}$ ) three times. The organic layer was combined and washed with brine $(30 \mathrm{~mL})$, then dried with sodium sulfate. After filtration, the solvent was evaporated in vacuo to obtain the oil crude product. The pure oil product was obtained by silica gel column chromatography using petrol ether/ethyl acetate (1:2, $v / v$ ). The yield of $N$-alpha-(tert-Butoxycarbonyl)- $N$-epsilon-1,8-naphthalimido-lysine (2) was $83.5 \%$. ${ }^{1} \mathrm{H}-\mathrm{NMR}\left(400 \mathrm{MHz}, \mathrm{CDCl}_{3}\right) \delta: 8.61(\mathrm{~d}, J=7.2 \mathrm{~Hz}, 2 \mathrm{H}$, naphthalene-H), $8.24(\mathrm{~d}, J=8.0 \mathrm{~Hz}$, $2 \mathrm{H}$, naphthalene-H), $7.77(\mathrm{t}, J=7.8 \mathrm{~Hz}, 2 \mathrm{H}$, naphthalene-H), $5.76(\mathrm{q}, J=5.12 \mathrm{~Hz}, 1 \mathrm{H}$, BocNHCH$)$, $4.51(\mathrm{~s}, 1 \mathrm{H}, \mathrm{BocNH}), 3.06\left(\mathrm{~d}, J=7.6 \mathrm{~Hz}, 2 \mathrm{H}, \mathrm{NCH}_{2}\right), 2.17-2.37\left(\mathrm{~m}, 2 \mathrm{H}, \mathrm{CHCH}_{2}\right), \mathrm{m}, 1.35$ (s, 9H, Boc), $1.30\left(\mathrm{t}, J=5.2 \mathrm{~Hz}, 2 \mathrm{H}, \mathrm{CHCH}_{2} \mathrm{CH}_{2}\right) .{ }^{13} \mathrm{C}-\mathrm{NMR}\left(100 \mathrm{MHz}, \mathrm{CDCl}_{3}\right) \delta: 164.00,134.51,131.99,131.77$, $128.52,127.17,122.34,53.11,40.42,29.77,28.61,28.48,23.74$.

\subsubsection{Synthesis of 3}

In a $100 \mathrm{~mL}$ flask, $N$-alpha-(tert-Butoxycarbonyl)- $N$-epsilon-1,8-naphthalimido-lysine (2) (1.50 g, $3.50 \mathrm{mmol})$, trimethylamine ( $0.95 \mathrm{~g}, 9.60 \mathrm{mmol})$, 4-dimethylaminopyridine (DMAP, $0.39 \mathrm{~g}, 3.20 \mathrm{mmol}$ ), and different diamines $(1.60 \mathrm{mmol})$ were dissolved in $50 \mathrm{~mL}$ methylene chloride. After stirring for $5 \mathrm{~min}, 1$-hydroxybenzotriazole (HOBt, $0.52 \mathrm{~g}, 3.85 \mathrm{mmol}$ ) was added into the mixture. The mixture was stirred in an ice-water bath for $0.5 \mathrm{~h}$. Then dicyclohexylcarbodiimide (DCC, $0.67 \mathrm{~g}, 3.50 \mathrm{mmol}$ ) was added into the mixture in batches. The mixture was warmed to room temperature and stirred overnight. Then, the mixture was washed with $5 \%$ citric acid solution $(50 \mathrm{~mL})$ and saturated sodium 
bicarbonate solution $(50 \mathrm{~mL})$. After drying with sodium sulfate, the solvent was evaporated in vacuo to obtain the crude product. After purification by silica gel column chromatography using petrol ether/ethyl acetate $(1: 3, v / v)$, the pure oil product was obtained.

Bis-(N-alpha-(tert-Butoxycarbonyl)-N-epsilon-1,8-naphthalimido-lysyl) ethylenediamine (3a). Yield 70.2\%. ${ }^{1} \mathrm{H}-\mathrm{NMR}\left(400 \mathrm{MHz}, \mathrm{CDCl}_{3}\right) \delta: 8.41(\mathrm{~d}, J=7.2 \mathrm{~Hz}, 4 \mathrm{H}$, naphthalene-H), $8.18(\mathrm{~d}, J=8.0 \mathrm{~Hz}, 4 \mathrm{H}$, naphthalene-H), $7.68(\mathrm{t}, J=7.8 \mathrm{~Hz}, 4 \mathrm{H}$, naphthalene- $\mathrm{H}), 7.02(\mathrm{~s}, 2 \mathrm{H}, \mathrm{CONH}), 5.66(\mathrm{t}, J=7.4 \mathrm{~Hz}$, $2 \mathrm{H}, \mathrm{BocNHCH}), 4.59(\mathrm{~s}, 2 \mathrm{H}, \mathrm{BocNH}), 3.32-3.49\left(\mathrm{~m}, 4 \mathrm{H}, \mathrm{CONHCH}_{2}\right), 3.01-3.06\left(\mathrm{~m}, 4 \mathrm{H}, \mathrm{NCH}_{2}\right)$, 2.27-2.28 (m, 4H, $\left.\mathrm{CHCH}_{2}\right), 1.46-1.57\left(\mathrm{~m}, 4 \mathrm{H}, \mathrm{NCH}_{2} \mathrm{CH}_{2}\right), 1.36(\mathrm{~s}, 18 \mathrm{H}, \mathrm{Boc}), 1.25$ (s, $\left.4 \mathrm{H}, \mathrm{CHCH}_{2} \mathrm{CH}_{2}\right)$. ${ }^{13} \mathrm{C}-\mathrm{NMR}\left(100 \mathrm{MHz}, \mathrm{CDCl}_{3}\right) \delta: 170.70,164.38,156.09,134.30,131.74,131.68,128.50,127.10,122.56,77.48$, 77.16, 76.85, 54.86, 40.26, 39.22, 29.91, 28.48, 28.16, 23.87. HR-MS $m / z$ (ESI), Calcd for $\mathrm{C}_{48} \mathrm{H}_{56} \mathrm{~N}_{6} \mathrm{O}_{10}$ $[\mathrm{M}+\mathrm{H}]^{+} 877.4141$, found 877.4095.

Bis-(N-alpha-(tert-Butoxycarbonyl)-N-epsilon-1,8-naphthalimido-lysyl)-1,4-butanediamin (3b). Yield 73.5\%. ${ }^{1} \mathrm{H}-\mathrm{NMR}\left(400 \mathrm{MHz}, \mathrm{CDCl}_{3}\right) \delta: 8.29(\mathrm{~d}, J=7.2 \mathrm{~Hz}, 4 \mathrm{H}$, naphthalene-H), $8.19(\mathrm{~d}, J=8.0 \mathrm{~Hz}, 4 \mathrm{H}$, naphthalene-H), $7.71(\mathrm{t}, J=7.8 \mathrm{~Hz}, 4 \mathrm{H}$, naphthalene-H), $6.32(\mathrm{~s}, 2 \mathrm{H}, \mathrm{CONH}), 5.53(\mathrm{~s}, 2 \mathrm{H}, \mathrm{BocNHCH})$, $4.55(\mathrm{~s}, 2 \mathrm{H}, \mathrm{BocNH}), 3.26-3.30\left(\mathrm{~m}, 4 \mathrm{H}, \mathrm{CONHCH}_{2}\right), 2.99-3.03\left(\mathrm{~m}, 4 \mathrm{H}, \mathrm{NCH}_{2}\right), 2.15(\mathrm{t}, J=7.6 \mathrm{~Hz}, 4 \mathrm{H}$, $\left.\mathrm{CHCH}_{2}\right), 1.42-1.52\left(\mathrm{~m}, 8 \mathrm{H}, \mathrm{CONHCH}_{2} \mathrm{CH}_{2}, \mathrm{NCH}_{2} \mathrm{CH}_{2}\right), 1.36(\mathrm{~s}, 18 \mathrm{H}, \mathrm{Boc}), 1.25(\mathrm{t}, J=7.2 \mathrm{~Hz}, 4 \mathrm{H}$, $\mathrm{CHCH}_{2} \mathrm{CH}_{2}$ ). ${ }^{13} \mathrm{C}-\mathrm{NMR}\left(100 \mathrm{MHz}, \mathrm{CDCl}_{3}\right)$ 8: 169.76, 164.34, 156.11, 134.37, 134.30, 131.87, 131.77, $131.69,128.48,127.17,127.12,122.55,77.48,77.16,76.84,54.81,40.27,39.58,29.79,28.49,28.13,26.65$, 23.85. HR-MS $m / z$ (ESI), Calcd for $\mathrm{C}_{50} \mathrm{H}_{60} \mathrm{~N}_{6} \mathrm{O}_{10}[\mathrm{M}+\mathrm{H}]^{+}$905.444, found 905.4350 .

Bis-(N-alpha-(tert-Butoxycarbonyl)-N-epsilon-1,8-naphthalimido-lysyl)-1,6-hexylenediamine (3c). Yield 71\%. ${ }^{1} \mathrm{H}-\mathrm{NMR}\left(400 \mathrm{MHz}, \mathrm{CDCl}_{3}\right) \delta: 8.55(\mathrm{~d}, J=7.2 \mathrm{~Hz}, 4 \mathrm{H}$, naphthalene- $\mathrm{H}), 8.22(\mathrm{~d}, J=8.0 \mathrm{~Hz}, 4 \mathrm{H}$, naphthalene-H), $7.75(\mathrm{t}, J=7.8 \mathrm{~Hz}, 4 \mathrm{H}$, naphthalene-H), $6.14(\mathrm{~s}, 2 \mathrm{H}, \mathrm{CONH}), 5.25(\mathrm{t}, J=6.88 \mathrm{~Hz}, 2 \mathrm{H}$, BocNHCH), 4.53 (s, 2H, BocNH), 2.59-3.02 (m, 8H, NCH $\left.{ }_{2}, \mathrm{CONHCH}_{2}\right), 2.11-2.15\left(\mathrm{~m}, 4 \mathrm{H}, \mathrm{CHCH}_{2}\right)$, 1.39-1.45 (m, 8H, $\left.\mathrm{CONHCH}_{2} \mathrm{CH}_{2}, \mathrm{NCH}_{2} \mathrm{CH}_{2}\right), 1.36(\mathrm{~s}, 18 \mathrm{H}, \mathrm{Boc}), 1.20-1.33\left(\mathrm{~m}, 4 \mathrm{H}, \mathrm{CHCH}_{2} \mathrm{CH}_{2}\right)$, 1.16-1.18 (m, 4H, CONHCH$\left.{ }_{2} \mathrm{CH}_{2} \mathrm{CH}_{2}\right) .{ }^{13} \mathrm{C}-\mathrm{NMR}\left(100 \mathrm{MHz}, \mathrm{CDCl}_{3}\right) \delta: 169.41,164.33,156.11,134.38$, 134.17, 131.81, 131.64, 131.60, 128.51, 127.15, 127.08, 122.73, 79.07, 54.42, 40.15, 37.89, 29.62, 28.50, 28.18, 24.45, 23.75. HR-MS $m / z$ (ESI), Calcd for $\mathrm{C}_{52} \mathrm{H}_{64} \mathrm{~N}_{6} \mathrm{O}_{10}[\mathrm{M}+\mathrm{H}]^{+} 933.4757$, found 933.4642 .

Bis-(N-alpha-(tert-Butoxycarbonyl)-N-epsilon-1,8-naphthalimido-lysyl)-1,4-phenylenedimethan-amine (3d). Yield 50.8\%. ${ }^{1} \mathrm{H}-\mathrm{NMR}\left(400 \mathrm{MHz}, \mathrm{CDCl}_{3}\right) \delta: 8.57(\mathrm{~d}, J=7.2 \mathrm{~Hz}, 4 \mathrm{H}$, naphthalene-H), $8.21(\mathrm{~d}, J=8.0 \mathrm{~Hz}$, $4 \mathrm{H}$, naphthalene-H), $7.74(\mathrm{t}, J=7.8 \mathrm{~Hz}, 4 \mathrm{H}$, naphthalene- $\mathrm{H}), 7.26(\mathrm{~s}, 2 \mathrm{H}$, benzene- $\mathrm{H}), 7.19(\mathrm{~s}, 2 \mathrm{H}$, benzene-H), $6.24(\mathrm{~s}, 2 \mathrm{H}, \mathrm{CONH}), 5.65(\mathrm{q}, J=5.52 \mathrm{~Hz}, 2 \mathrm{H}, \mathrm{BocNHCH}), 4.51(\mathrm{~s}, 2 \mathrm{H}, \mathrm{BocNH}), 4.37-4.48(\mathrm{~m}$, $4 \mathrm{H}$, xylyl-H), 2.97-2.31 (m, 4H, NCH$)_{2}, 2.23-2.31\left(\mathrm{~m}, 4 \mathrm{H}, \mathrm{CHCH}_{2}\right), 1.48\left(\mathrm{t}, J=5.2 \mathrm{~Hz}, 4 \mathrm{H}, \mathrm{NCH}_{2} \mathrm{CH}_{2}\right)$, 1.35 (s, 18H, Boc), 1.25 (s, 4H, $\left.\mathrm{CHCH}_{2} \mathrm{CH}_{2}\right) .{ }^{13} \mathrm{C}-\mathrm{NMR}\left(100 \mathrm{MHz}, \mathrm{CDCl}_{3}\right)$ 8: 169.52, 164.38, 156.07, 137.52, 134.49, 131.97, 131.71, 128.49, 128.17, 127.19, 122.41, 54.82, 43.57, 40.22, 29.82, 28.56, 28.47, 28.20, 23.80. HR-MS $m / z$ (ESI), Calcd for $\mathrm{C}_{54} \mathrm{H}_{60} \mathrm{~N}_{6} \mathrm{O}_{10}[\mathrm{M}+\mathrm{Na}]^{+}$975.4263, found 975.4187.

Bis-(N-alpha-(tert-Butoxycarbonyl)-N-epsilon-1,8-naphthalimido-lysyl)-1,3-phenylenedimethan-amine (3e). Yield 74.1\%. ${ }^{1} \mathrm{H}-\mathrm{NMR}\left(400 \mathrm{MHz}, \mathrm{CDCl}_{3}\right) \delta: 8.46(\mathrm{~d}, \mathrm{~J}=7.2 \mathrm{~Hz}, 4 \mathrm{H}$, naphthalene-H), $8.08(\mathrm{~d}, J=8.0 \mathrm{~Hz}, 4 \mathrm{H}$, naphthalene-H), $7.61(\mathrm{t}, J=7.8 \mathrm{~Hz}, 4 \mathrm{H}$, naphthalene-H), $7.29(\mathrm{~s}, 1 \mathrm{H}$, benzene-H), $7.26(\mathrm{~s}, 1 \mathrm{H}$, benzene-H), $7.13(\mathrm{~d}, J=7.88 \mathrm{~Hz}, 2 \mathrm{H}$, benzene-H), $6.54(\mathrm{~s}, 2 \mathrm{H}, \mathrm{CONH})$, $5.66(\mathrm{t}, J=7.4,2 \mathrm{H}, \mathrm{BocNHCH}), 4.62(\mathrm{~s}, 2 \mathrm{H}, \mathrm{BocNH}), 4.37-4.55(\mathrm{~m}, 4 \mathrm{H}, \mathrm{xylyl}-\mathrm{H}), 3.00-3.06(\mathrm{~m}, 4 \mathrm{H}$, $\left.\mathrm{NCH}_{2}\right), 2.25-2.31\left(\mathrm{~m}, 4 \mathrm{H}, \mathrm{CHCH}_{2}\right), 1.51\left(\mathrm{t}, J=5.8 \mathrm{~Hz}, 4 \mathrm{H}, \mathrm{NCH}_{2} \mathrm{CH}_{2}\right), 1.48\left(\mathrm{~s}, 4 \mathrm{H}, \mathrm{CHCH}_{2} \mathrm{CH}_{2}\right), 1.37(\mathrm{~s}$, 18H, Boc). ${ }^{13} \mathrm{C}-\mathrm{NMR}\left(100 \mathrm{MHz}, \mathrm{CDCl}_{3}\right) \delta: 169.64,164.30,156.12,138.93,134.22,131.70,131.52,128.79$, 128.29, 127.03, 126.96, 126.65, 122.33, 79.04, 77.48, 77.16, 76.84, 54.88, 43.57, 40.27, 29.82, 28.47, 28.30, 23.92. HR-MS $m / z$ (ESI), Calcd for $\mathrm{C}_{54} \mathrm{H}_{60} \mathrm{~N}_{6} \mathrm{O}_{10}[\mathrm{M}+\mathrm{Na}]^{+} 975.4263$, found 975.4223 .

\subsubsection{Synthesis of 4}

In a $100 \mathrm{~mL}$ flask, compound $3(0.25 \mathrm{mmol})$ was dissolved in $15 \mathrm{~mL}$ ethanol. A quantity of $30 \mathrm{~mL}$ $\mathrm{HCl}$ ethanol solution was added under stirring. The mixture was stirred at room temperature overnight. 
The solvent was evaporated in vacuo to obtain the crude product. After washing with methyl tert-butyl ether, a white powder was obtained.

Bis-(N-epsilon-1,8-naphthalimido-lysyl) ethylenediamine (4a). Yield 85.5\%. ${ }^{1} \mathrm{H}-\mathrm{NMR}\left(400 \mathrm{MHz}_{2} \mathrm{D}_{2} \mathrm{O}\right)$ $\delta: 8.21(\mathrm{~d}, J=7.2 \mathrm{~Hz}, 4 \mathrm{H}$, naphthalene-H), $8.07(\mathrm{~d}, J=8.0 \mathrm{~Hz}, 4 \mathrm{H}$, naphthalene- $\mathrm{H}), 7.58(\mathrm{t}, J=7.8 \mathrm{~Hz}$, $4 \mathrm{H}$, naphthalene-H), $5.53(\mathrm{q}, J=5.04 \mathrm{~Hz}, 2 \mathrm{H}, \mathrm{BocNHCH}), 3.23-3.36\left(\mathrm{~m}, 4 \mathrm{H}, \mathrm{NCH}_{2}\right), 2.93(\mathrm{t}, J=7.8 \mathrm{~Hz}$, $\left.4 \mathrm{H}, \mathrm{CHCH}_{2}\right), 1.97-2.23\left(\mathrm{~m}, 4 \mathrm{H}, \mathrm{NCH}_{2} \mathrm{CH}_{2}\right), 1.49-1.71\left(\mathrm{~m}, 4 \mathrm{H}, \mathrm{CHCH}_{2} \mathrm{CH}_{2}\right), 1.32-1.34(\mathrm{~m}, 4 \mathrm{H}$, $\left.\mathrm{CONHCH}_{2} \mathrm{CH}_{2}\right) .{ }^{13} \mathrm{C}-\mathrm{NMR}\left(100 \mathrm{MHz}, \mathrm{D}_{2} \mathrm{O}\right) \delta: 175.20,165.27,135.51,131.67,131.08,127.41,126.97$, 120.76, 54.02, 39.37, 39.16, 29.58, 27.21, 26.43, 22.66, 16.77. HR-MS m/z (ESI), Calcd for $\mathrm{C}_{38} \mathrm{H}_{40} \mathrm{~N}_{6} \mathrm{O}_{6}$ $[\mathrm{M}+\mathrm{H}]^{+} 677.3082$, found 677.3036 .

Bis-(N-epsilon-1,8-naphthalimido-lysyl)-1,4-butanediamin (4b). Yield 79.2\%. ${ }^{1} \mathrm{H}-\mathrm{NMR}\left(400 \mathrm{MHz}, \mathrm{D}_{2} \mathrm{O}\right)$ $\delta: 8.34(\mathrm{~d}, J=7.2 \mathrm{~Hz}, 4 \mathrm{H}$, naphthalene-H), $8.08(\mathrm{~d}, J=8.0 \mathrm{~Hz}, 4 \mathrm{H}$, naphthalene- $\mathrm{H}), 7.62(\mathrm{t}, J=7.8 \mathrm{~Hz}, 4 \mathrm{H}$, naphthalene-H), $5.56(\mathrm{q}, J=5.04 \mathrm{~Hz}, 2 \mathrm{H}, \mathrm{BocNHCH}), 3.19\left(\mathrm{~s}, 4 \mathrm{H}, \mathrm{NCH}_{2}\right), 2.93\left(\mathrm{t}, J=7.8 \mathrm{~Hz}, 4 \mathrm{H}, \mathrm{CHCH}_{2}\right)$, 2.07-2.22 (m, 4H, NCH $\left.\mathrm{CH}_{2}\right), 1.68-1.72\left(\mathrm{~m}, 4 \mathrm{H}, \mathrm{CHCH}_{2} \mathrm{CH}_{2}\right), 1.53-1.55\left(\mathrm{~m}, 4 \mathrm{H}, \mathrm{CONHCH}_{2} \mathrm{CH}_{2}\right)$, 1.21-1.54 (m, 4H, $\left.\mathrm{CH}_{2} \mathrm{CH}_{2}\right) .{ }^{13} \mathrm{C}-\mathrm{NMR}\left(100 \mathrm{MHz}, \mathrm{D}_{2} \mathrm{O}\right) \delta: 172.39,171.06,165.59,135.68,131.94,130.93$, 127.24, 127.04, 120.61, 54.07, 42.79, 39.12, 38.91, 27.27, 26.39, 25.89, 25.68, 22.59. HR-MS $m / z$ (ESI), Calcd for $\mathrm{C}_{40} \mathrm{H}_{44} \mathrm{~N}_{6} \mathrm{O}_{6}[\mathrm{M}+\mathrm{H}]^{+} 705.3395$, found found 705.3382 .

Bis-(N-epsilon-1,8-naphthalimido-lysyl)-1,6-hexylenediamine (4c). Yield 83.6\%. ${ }^{1} \mathrm{H}-\mathrm{NMR}\left(400 \mathrm{MHz}, \mathrm{D}_{2} \mathrm{O}\right)$ $\delta: 8.17(\mathrm{~d}, J=7.2 \mathrm{~Hz}, 4 \mathrm{H}$, naphthalene-H), $8.05(\mathrm{~d}, J=8.0 \mathrm{~Hz}, 4 \mathrm{H}$, naphthalene- $\mathrm{H}), 7.49(\mathrm{t}, J=7.8 \mathrm{~Hz}, 4 \mathrm{H}$, naphthalene-H), 5.20 (q, $J=5.6 \mathrm{~Hz}, 2 \mathrm{H}, \mathrm{BocNHCH}), 2.78-2.92\left(\mathrm{~m}, 4 \mathrm{H}, \mathrm{NCH}_{2}, \mathrm{CHCH}_{2}\right), 1.89-2.13(\mathrm{~m}$, $\left.4 \mathrm{H}, \mathrm{NCH}_{2} \mathrm{CH}_{2}\right), 1.61-1.70\left(\mathrm{~m}, 4 \mathrm{H}, \mathrm{CHCH}_{2} \mathrm{CH}_{2}\right), 1.23-1.35\left(\mathrm{~m}, 4 \mathrm{H}, \mathrm{NHCH}_{2} \mathrm{CH}_{2}\right), 1.05(\mathrm{~d}, J=5.2 \mathrm{~Hz}, 4 \mathrm{H}$, $\left.\mathrm{CONHCH}_{2} \mathrm{CH}_{2}\right), 0.80\left(\mathrm{t}, J=6.2 \mathrm{~Hz}, 4 \mathrm{H}, \mathrm{CONHCH}_{2} \mathrm{CH}_{2} \mathrm{CH}_{2}\right) .{ }^{13} \mathrm{C}-\mathrm{NMR}\left(100 \mathrm{MHz}, \mathrm{D}_{2} \mathrm{O}\right) \delta: 170.96$, 165.32, 135.37, 131.41, 130.97, 127.26, 126.94, 120.86, 54.16, 39.17, 38.41, 28.09, 27.52, 26.46, 24.89, 22.85. HR-MS $m / z$ (ESI), Calcd for $\mathrm{C}_{42} \mathrm{H}_{48} \mathrm{~N}_{6} \mathrm{O}_{6}[\mathrm{M}+\mathrm{H}]^{+}$733.3708, found 733.3665.

Bis-(N-epsilon-1,8-naphthalimido-lysyl)-1,4-phenylenedimethan-amine (4d). Yield 78.9\%. ${ }^{1} \mathrm{H}-\mathrm{NMR}(400 \mathrm{MHz}$, $\left.\mathrm{D}_{2} \mathrm{O}\right) \delta:$ 7.77-7.88 (m, 4H, naphthalene-H), 7.50-7.77 (m, 4H, naphthalene-H), 7.25-7.49 (m, 4H, naphthalene-H), 6.98-7.04 (m, 4H, benzene-H), $7.02(\mathrm{~m}, 4 \mathrm{H}$, benzene- $\mathrm{H}), 5.37(\mathrm{t}, J=7.0 \mathrm{~Hz}, 2 \mathrm{H}, \mathrm{BocNHCH})$, 3.85-4.45 (m, 4H, xylyl-H), 2.92-2.98 (m, 4H, NCH $), 1.92-2.24\left(\mathrm{~m}, 4 \mathrm{H}, \mathrm{CHCH}_{2}\right), 1.67-1.77(\mathrm{~m}, 4 \mathrm{H}$, $\left.\mathrm{NCH}_{2} \mathrm{CH}_{2}\right), 1.26-1.39\left(\mathrm{~m}, 4 \mathrm{H}, \mathrm{CHCH}_{2} \mathrm{CH}_{2}\right) .{ }^{13} \mathrm{C}-\mathrm{NMR}\left(100 \mathrm{MHz}, \mathrm{CDCl}_{3}\right) \delta: 171.33,165.03,137.90,135.17$, $131.10,130.50,128.43,126.73,126.65,120.23,57.41,54.21,43.02,39.22,27.63,26.54,25.89,23.03$. HR-MS $m / z$ (ESI), Calcd for $\mathrm{C}_{44} \mathrm{H}_{44} \mathrm{~N}_{6} \mathrm{O}_{6}[\mathrm{M}+\mathrm{H}]^{+} 753.3395$, found 753.3312 .

Bis-(N-epsilon-1,8-naphthalimido-lysyl)-1,3-phenylenedimethan-amine (4e). Yield 88.9. ${ }^{1} \mathrm{H}-\mathrm{NMR}(400 \mathrm{MHz}$, $\left.\mathrm{D}_{2} \mathrm{O}\right) \delta: 7.88(\mathrm{~d}, J=7.2 \mathrm{~Hz}, 4 \mathrm{H}$, naphthalene-H), $7.77(\mathrm{~d}, J=8.0 \mathrm{~Hz}, 4 \mathrm{H}$, naphthalene-H), $7.24(\mathrm{t}, J=7.8 \mathrm{~Hz}, 4 \mathrm{H}$, naphthalene-H), 6.98-7.04 (m, 4H, benzene-H), $5.35(\mathrm{q}, J=6.04 \mathrm{~Hz}, 2 \mathrm{H}$, BocNHCH), 4.05-4.23 (q, $J=15.04 \mathrm{~Hz}, 4 \mathrm{H}, \mathrm{xylyl}-\mathrm{H}), 2.97\left(\mathrm{t}, J=7.6 \mathrm{~Hz}, 4 \mathrm{H}, \mathrm{NCH}_{2}\right), 1.94-2.22(\mathrm{~m}, 4 \mathrm{H}$, $\left.\mathrm{CHCH}_{2}\right), 1.69-1.76\left(\mathrm{~m}, 4 \mathrm{H}, \mathrm{NCH}_{2} \mathrm{CH}_{2}\right), 1.32-1.39\left(\mathrm{~m}, 4 \mathrm{H}, \mathrm{CHCH}_{2} \mathrm{CH}_{2}\right) .{ }^{13} \mathrm{C}-\mathrm{NMR}\left(100 \mathrm{MHz}_{2} \mathrm{CDCl}_{3}\right)$ $\delta: 171.33,165.03,137.90,135.17,131.10,130.50,128.43,126.73,126.65,120.23,57.41,54.21,43.02,39.22$, 27.63, 26.54, 25.89, 23.03. HR-MS m/z (ESI), Calcd for $\mathrm{C}_{44} \mathrm{H}_{44} \mathrm{~N}_{6} \mathrm{O}_{6}[\mathrm{M}+\mathrm{H}]^{+}$753.3395, found 753.3358 .

\subsection{UV-Vis Titration}

A quantity of $20 \mu \mathrm{L}$ stock solution of each bis-naphthalimide compound $4 \mathbf{a}-\mathbf{e}(5 \mathrm{mM})$ was diluted with $3 \mathrm{~mL}$ phosphate buffer $(100 \mathrm{mM}, \mathrm{pH}=7.4)$. An increasing volume of CT-DNA solution was added into the solution. Then, the solution was stirred and incubated at $25{ }^{\circ} \mathrm{C}$ for $10 \mathrm{~min}$. The UV-Vis spectra in the absence and presence of DNA were recorded using a Shimadzu UV-2501 spectrophotometer. The binding constant $K_{b}$ was calculated from a $D / \Delta \varepsilon_{a p p}$ vs. $D$ plot according to the following equation [31]: 


$$
D / \Delta \varepsilon_{a p p}=D / \Delta \varepsilon+1 /\left[(\Delta \varepsilon) K_{b}\right]
$$

where $D$ is the concentration of DNA, $\Delta \varepsilon_{a p p}=\left[\varepsilon_{A}-\varepsilon_{F}\right], \varepsilon_{\mathrm{A}}=A_{\text {obs }} /$ [compound], $\Delta \varepsilon=\left[\varepsilon_{B}-\varepsilon_{F}\right]$, and $\varepsilon_{B}$ and $\varepsilon_{F}$ correspond to the extinction coefficients of the DNA-compound adduct and unbound compound, respectively.

\subsection{Viscosity Study}

Viscometric titration was performed at $25{ }^{\circ} \mathrm{C}$ using an Ubblehode viscometer. A quantity of $5 \mathrm{~mL}$ CT DNA (2.8 mM) was diluted with $20 \mathrm{~mL}$ phosphate buffer (50.0 mM, pH 7.4). The different flow time was measured using a stopwatch while varying the concentrations of compounds. The plot of $\left(\eta / \eta_{0}\right)^{1 / 3}$ vs. $r$ was obtained according to the flow time, where $\eta$ and $\eta_{0}$ are the flow time of the presence and absence of compounds, respectively, and $r$ is equal to [compound]/[DNA].

\subsection{Cytotoxicity Assay}

Human gastric carcinoma cell line (BGC823) and human esophageal carcinoma cell line (EC109) were cultured in RPMI 1640 medium supplemented with 10\% fetal bovine serum, $100 \mathrm{units} / \mathrm{mL}$ penicillin, and 100 units $/ \mathrm{mL}$ penicillin streptomycin. The cell culture was kept in 5\% $\mathrm{CO}_{2}$ under humidified conditions at $37^{\circ} \mathrm{C}$. The culture solution was changed every other day, and the subcultures were performed with $0.25 \%$ trypsin. The compounds were solubilized in RPMI 1640 medium and diluted to different concentrations immediately prior to use.

The cytotoxicity of bis-naphthalimide compounds was assessed using the thiazolyl blue tetrazolium bromide (MTT) assay in vitro. Tumor cells were planted into 96-well microtiter plates at a density of $5.0 \times 10^{4}$ cells / well. After being cultured in $5 \% \mathrm{CO}_{2}$ under humidified conditions at $37^{\circ} \mathrm{C}$ for $24 \mathrm{~h}$, various compound medium solutions were added to obtain the final drug concentrations of $0,12.5,25,50,100$, and $200 \mu \mathrm{mol} / \mathrm{L}$, respectively. After incubation for $24 \mathrm{~h}, 10 \mu \mathrm{L} \mathrm{MTT}(5 \mathrm{mg} / \mathrm{L})$ was added to each cell and the mixture was incubated for $4 \mathrm{~h}$. Then, the medium was removed and replaced by $150 \mu \mathrm{L}$ DMSO to solubilize the converted purple dye in the culture plates. The absorbance of each cell was measured using a Bio-rad 680 microplate reader (Hercules, CA, USA) at $490 \mathrm{~nm}$. The $\mathrm{IC}_{50}$, which inhibits the growth of $50 \%$ of cells relative to nontreated control cells, was calculated as the concentration of the tested compound by linear fitting.

\subsection{Morphology Observation}

Tumor cells were planted into 24-well microtiter plates at a density of $5.0 \times 10^{6}$ cells/well. After incubation for $24 \mathrm{~h}$, compound solution was added and incubated for $48 \mathrm{~h}$. Morphological changes of the cells were observed using light microscopy.

\section{Conclusions}

In this paper, a series of bis-naphthalimide derivatives with different diamine linkers were designed and synthesized. The DNA binding constants and binding modes of the compounds were measured by UV-Vis titration and viscosity experiments. The results showed that the length of diamine linkers significantly influenced the binding ability of the bis-naphthalimide derivatives. The compounds with shorter linkers showed larger binding constants and the classical binding mode. However, in cytotoxicity assay experiments, the compound $\mathbf{4 d}$ with the rigid $p$-xylylenediamine linker showed better cytotoxicity than did the other bis-naphthalimide derivatives. The morphology observation of BGC823 cell line incubated with $4 \mathrm{~d}$ also exhibited the inhibition of cancer cell growth.

Acknowledgments: This work was financially supported by the National Science Foundation of China (No. 21362026) and Young Talent Program of NingXia medical University. 
Author Contributions: Yu Huang and Yan-Ru Fan design this work and analysis the data. Min Huang, Da-Nian Tian and Xin-Bin Yang play important role in analysis and interpretation of data. Chun-Xia Wu and Yu Song collected all the experiment data.

Conflicts of Interest: The authors declare no conflict of interest.

\section{References}

1. Braña, M.F.; Ramos, A. Naphthalimides as anti-cancer agents: Synthesis and biological activity. Curr. Med. Chem. Anticancer Agents 2001, 1, 237-255. [CrossRef] [PubMed]

2. Gellerman, G. Recent Developments in the Synthesis and Applications of Anticancer Amonafide Derivatives. A Mini Review. Lett. Drug Des. Discov. 2016, 13, 47-63. [CrossRef]

3. Banerjee, S.; Veale, E.B.; Phelan, C.M.; Murphy, S.A.; Tocci, G.M.; Gillespie, L.J.; Frimannsson, D.O.; Kelly, J.M.; Gunnlaugsson, T. Recent advances in the development of 1,8-naphthalimide based DNA targeting binders, anticancer and fluorescent cellular imaging agents. Chem. Soc. Rev. 2013, 42, 1601-1618. [CrossRef] [PubMed]

4. Norton, J.T.; Witschi, M.A.; Luong, L.; Kawamura, A.; Ghosh, S.; Stack, M.S.; Sim, E.; Avram, M.J.; Appella, D.H.; Huang, S. Synthesis and anticancer activities of 6-amino amonafide derivatives. Anticancer Drugs 2008, 19, 23-36. [CrossRef] [PubMed]

5. Lv, M.; Xu, H. Overview of naphthalimide analogs as anticancer agents. Curr. Med. Chem. 2009, 16, 4797-4813. [CrossRef] [PubMed]

6. Freeman, C.L.; Swords, R.; Giles, F.J. Amonafide: A future in treatment of resistant and secondary acute myeloid leukemia? Expert Rev. Hematol. 2012, 5, 17-26. [CrossRef] [PubMed]

7. Stone, R.M.; Mazzola, E.; Neuberg, D.; Allen, S.L.; Pigneux, A.; Stuart, R.K.; Wetzler, M.; Rizzieri, D.; Erba, H.P.; Damon, L.; et al. Phase III open-label randomized study of cytarabine in combination with amonafide L-malate or daunorubicin as induction therapy for patients with secondary acute myeloid leukemia. J. Clin. Oncol. 2015, 33, 1252-1257. [CrossRef] [PubMed]

8. Lu, Y.T.; Chen, T.L.; Chang, K.S.; Chang, C.M.; Wei, T.Y.; Liu, J.W.; Hsiao, C.A.; Shih, T.L. Synthesis of novel C4-benzazole naphthalimide derivatives with potent anti-tumor properties against murine melanoma. Bioorg. Med. Chem. 2017, 25, 789-794. [CrossRef] [PubMed]

9. Wang, Y.; Zhang, J.; Li, M.; Li, M.; Xie, S.; Wang, C. Synthesis and evaluation of novel amonafide-polyamine conjugates as anticancer agents. Chem. Biol. Drug Des. 2017, 89, 670-680. [CrossRef] [PubMed]

10. Ge, C.C.; Chang, L.P.; Zhao, Y.; Chang, C.C.; Xu, X.J.; He, H.Y.; Wang, Y.X.; Dai, F.J.; Xie, S.Q.; Wang, C.J. Design, Synthesis and Evaluation of Naphthalimide Derivatives as Potential Anticancer Agents for Hepatocellular Carcinoma. Molecules 2017, 22, 342. [CrossRef] [PubMed]

11. Brider, T.; Redko, B.; Oron-Herman, M.; Cohen-Matzlich, A.; Gerlitz, G.; Gellerman, G.; Grynszpan, F. Synthesis and in vitro anticancer evaluation of 1,8-naphthalimide $N(4)$ and $S(4)$-derivatives combining DNA intercalation and alkylation capabilities. Res. Chem. Intermed. 2016, 42, 1741-1757. [CrossRef]

12. Bousquet, P.F.; Braña, M.F.; Conlon, D.; Fitzgerald, K.M.; Perron, D.; Cocchiaro, C.; Miller, R.; Moran, M.; George, J.; Qian, X.D.; et al. Preclinical evaluation of LU 79553: A novel bis-naphthalimide with potent antitumor activity. Cancer Res. 1995, 55, 1176-1180. [PubMed]

13. Kamal, A.; Ramu, R.; Tekumalla, V.; Ramesh Khanna, G.B.; Zingde, S.M. Remarkable DNA binding affinity and potential anti-cancer activity of pyrrolo[2,1-c][1,4] benzodiazepine-naphthalimide conjugates linked through piperazine side-armed alkane spacers. Bioorg. Med. Chem. 2008, 16, 7218-7224. [CrossRef] [PubMed]

14. Veale, E.B.; Frimannsson, D.O.; Lawler, M.; Gunnlaugsson, T. 4-Amino-1,8-naphthalimide-based Tröger's bases as high affinity DNA targeting fluorescent supramolecular scaffolds. Org. Lett. 2009, 11, 4040-4043. [CrossRef] [PubMed]

15. Veale, E.B.; Gunnlaugsson, T. Synthesis, photophysical, and DNA binding studies of fluorescent Tröger's base derived 4-amino-1,8-naphthalimide supramolecular clefts. J. Org. Chem. 2010, 75, 5513-5525. [CrossRef] [PubMed]

16. Antonini, I.; Santoni, G.; Lucciarini, R.; Amantini, C.; Sparapani, S.; Magnano, A. Synthesis and biological evaluation of new asymmetrical bisintercalators as potential antitumor drugs. J. Med. Chem. 2006, 49, 7198-7207. [CrossRef] [PubMed] 
17. Ott, I.; Xu, Y.; Qian, X. Fluorescence properties and antiproliferative effects of mono-, bis-, and tris-thiophenylnaphthalimides: Results of a comparative pilot study. J. Photochem. Photobiol. B 2011, 105, 75-80. [CrossRef] [PubMed]

18. Suzuki, K.; Nagasawa, H.; Uto, Y.; Sugimoto, Y.; Noguchi, K.; Wakida, M.; Wierzba, K.; Terada, T.; Asao, T.; Yamada, Y.; et al. Napthalimidobenzamide DB-51630: A novel DNA binding agent inducing p300 gene expression and exerting a potent anti-cancer activity. Bioorg. Med. Chem. 2005, 13, 4014-4021. [CrossRef] [PubMed]

19. Pavlov, V.; Kong Thoo Lin, P.; Rodilla, V. Cytotoxicity, DNA binding and localisation of novel bis-naphthalimidopropyl polyamine derivatives. Chem. Biol. Interact. 2001, 137, 15-24. [CrossRef]

20. Lin, P.K.; Pavlov, V.A. The synthesis and in vitro cytotoxic studies of novel bis-naphthalimidopropyl polyamine derivatives. Bioorg. Med. Chem. Lett. 2000, 10, 1609-1612. [CrossRef]

21. Barron, G.A.; Bermano, G.; Gordon, A.; Kong Thoo Lin, P. Synthesis, cytotoxicity and DNA-binding of novel bisnaphthalimidopropyl derivatives in breast cancer MDA-MB-231 cells. Eur. J. Med. Chem. 2010, 45, 1430-1437. [CrossRef] [PubMed]

22. Filosa, R.; Peduto, A.; Micco, S.D.; Caprariis, P.; Festa, M.; Petrella, A.; Capranico, G.; Bifulco, G. Molecular modelling studies, synthesis and biological activity of a series of novel bis-naphthalimides and their development as new DNA topoisomerase II inhibitors. Bioorg. Med. Chem. 2009, 17, 13-24. [CrossRef] [PubMed]

23. Yang, P.; Yang, Q.; Qian, X.B. Novel DNA bis-intercalators of isoquinolino[4,5-bc]acridines: Design, synthesis and evaluation of cytotoxic activity. Tetrahedron 2005, 61, 11895-11901. [CrossRef]

24. Ralton, L.D.; Bestwick, C.S.; Milne, L.; Duthie, S.; Kong Thoo Lin, P. Bisnaphthalimidopropyl spermidine induces apoptosis within colon carcinoma cells. Chem. Biol. Interact. 2009, 177, 1-6. [CrossRef] [PubMed]

25. Rong, R.X.; Sun, Q.; Ma, C.L.; Chen, B.; Wang, W.Y.; Wang, Z.A.; Wang, K.R.; Cao, Z.R.; Li, X.L. Development of novel bis-naphthalimide derivatives and their anticancer properties. Med. Chem. Commun. 2016, 7, 679-685. [CrossRef]

26. Wu, A.; Xu, Y.; Qian, X. Novel naphthalimide-amino acid conjugates with flexible leucine moiety as side chain: Design, synthesis and potential antitumor activity. Bioorg. Med. Chem. 2009, 17, 592-599. [CrossRef] [PubMed]

27. Yang, Q.; Yang, P.; Qian, X.; Tong, L. Naphthalimide intercalators with chiral amino side chains: Effects of chirality on DNA binding, photodamage and antitumor cytotoxicity. Bioorg. Med. Chem. Lett. 2008, 18, 6210-6213. [CrossRef] [PubMed]

28. McMasters, S.; Kelly, L.A. Sequence-dependent interactions of cationic naphthalimides and polynucleotides. Photochem. Photobiol. 2007, 83, 889-896. [CrossRef] [PubMed]

29. Lerman, L.S. Structural considerations in the interaction of DNA and acridines. J. Mol. Biol. 1961, 3, 18-30. [CrossRef]

30. Satyanarayana, S.; Dabrowiak, J.C.; Chaires, J.B. Tris(phenanthroline)ruthenium(II) enantiomer interactions with DNA: Mode and specificity of binding. Biochemistry 1993, 32, 2573-2584. [CrossRef] [PubMed]

31. Kumar, C.V.; Asuncion, E.H. DNA Binding Studies and Sites Fluorescence Sensitization of an Anthryl Probe. J. Am. Chem. Soc. 1993, 115, 8547-8553. [CrossRef]

Sample Availability: Samples of the compounds 1-4 are available from the authors. 